\title{
Connected Layers: Evaluating Visualizations of Embodiment in Contemporary Dance Performances
}

\author{
NUNO N. CORREIA, University of Greenwich, UK and ITI/LARSyS, Portugal \\ RAUL MASU, ITI/LARSyS, Portugal and DI, FCT, Universidade Nova de Lisboa, Portugal \\ AN HOANG DIEU PHAM, Erasmus University Rotterdam, Netherlands \\ JOCHEN FEITSCH, University of Applied Sciences Düsseldorf, Germany
}

There has been a growing interest in interactive visuals in contemporary dance performances. These visuals often rely on embodied interaction techniques, such as motion capture or biosignal sensors. However, there is a lack of research into how audience members experience these interactive visuals, and how to enhance that experience. We conducted an audience study, involving four different dance performances. Each of the performances explored a different approach for interaction involving visuals. We collected data from audience members, regarding their experience of the performances, using questionnaires and interviews. The analysis of this data allows us to identify implications for design: balancing trade-offs within a mapping clarity spectrum; connecting layers; visuals as co-creative mediator; defined territory and individuality of visuals; exploration of perspective shift and abstract fragmentation. We argue that these considerations are relevant for designers of systems visualizing embodied interaction, not just for dance, but also for other related applications.

CCS Concepts: • Human-centered computing $\rightarrow$ Empirical studies in HCI; HCI theory, concepts and models; • Applied computing $\rightarrow$ Performing arts.

Additional Key Words and Phrases: Audience Study; Interactive Visuals; Contemporary Dance; Performance Arts; Motion Capture; Biosignal Sensors

\section{INTRODUCTION}

Interactive visuals have become a common component of contemporary dance performances. In particular, interactive visuals, mapped to information extracted from the dancer's body and movements, have been explored by numerous artists. Some notable examples are the work of Wechsler and Weiss [40], OpenEndedGroup [15], Troika Ranch [14], the Reactor for Awareness in Motion project [23], and Fdili Alaoui [16, 18]. Recent research has attempted to provide design insights regarding these systems. Masu and colleagues [27] conducted a study with a group of ten contemporary dance artists working with technology, to shed light regarding how they would wish to use interactive technology, with an emphasis on interactive visuals, resulting in design guidelines [27]. This aligns with an increased attention to interactive visuals in performance art in general, particularly in electronic music performances [13]. In recent years, 
there have been a number of studies analyzing how audiences perceive and value the inclusion of interactive visuals in performance. However, these studies have mostly focused on music performances (e.g [5, 11]), not contemporary dance.

According to Wechsler et al. [40], there are four levels of interaction between a human and a computational system in dance performances: 1) a purely technical level, when dancer data is successfully mapped to a parameter in the system; 2) performer awareness of affecting their environment through the system; 3) audience awareness of the interaction between dancer and system; and 4) audience interaction with the system. Wechsler et al. identify great potential in the third level: "if an art work and its technology are prepared carefully enough, then the interactivity can be accessible to an audience directly and this is the greatest artistic potential" [40]. We argue that audience studies are needed to understand what design approaches can lead to this "accessibility" of interactivity with visuals to audiences in dance performances. However, there is a lack of such studies. This leads to our research question: What design approaches for interactive visuals in dance can lead to an engaging and aesthetically appealing experience for the audience?

To answer our research question, we adopted an 'in the wild' approach, informed by perspectives from Benford et al. [2]: we set up a public event in Tanzhaus NRW, a prominent dance venue in Düsseldorf, in October 2019. The event consisted of four short contemporary dance performances (around 15 minutes each), previously and iteratively developed in the scope of the project Moving Digits ${ }^{1}$. Each of the performances followed a different interaction design approach, to obtain information from the body and map it to visuals, based on: motion capture (mocap) suit; sensors (breath, positional and color); video camera; and minimal interaction. After each performance, audience members were asked to fill in a questionnaire composed of a set of four Likert scale questions and two open-ended questions, repeated for each performance. In the end of the event, we conducted interviews with four audience members. Our study is therefore situated in the territory of performance-led research in the wild, in which "artists drive the creation of novel performances with the support of HCI researchers that are then deployed and studied at public performance" [2].

We have previously reported on the quantitative side of the questionnaire [26]. That had allowed us to preliminarily identify which performances succeeded or failed in terms of a few specific aspects, but did not provide any deep reasoning behind that. In this paper, we follow an additional qualitative multi-method approach: we analyze 1) the open-ended responses of the questionnaire and 2) the interviews. We argue that the results and our conclusions can be useful for designers and artists aiming to visualize body data in dance. Additionally, we propose that our findings can also be applied to other fields (such as other areas in arts and entertainment).

\section{RELATED WORK}

\subsection{Interactive Visuals in Performance}

Performance arts have increasingly been adopting interactive visuals for the stage. Art forms such as music, theatre, opera and contemporary dance have been exploring the inclusion of interactive visuals. The emergence of "screen-based performance" based on digital computation, particularly from the 1990s, has adopted "a long litany of names such as audiovisual performance, real-time video, live cinema, performance cinema, and VJ culture" [34]. We will use the term 'interactive visuals' to refer to visuals for the stage manipulated in real-time. This emergence was due to breakthroughs in computation, particularly "the development of hardware and software components for the capture, processing, and manipulation of image" [34]. In electronic music contexts, interactive visuals can assist audiences toward an increased understanding of performers' actions [12]. In theatre, interactive visuals can complement the story and allow audiences

\footnotetext{
${ }^{1}$ https://movingdigits.eu
} 
to "focus attention on the connection between performer and digital environment" [7]. Regarding opera, Machover's Death and the Powers is an example of use of interactive visuals to mirror and amplify performers' gestures [38].

In dance, "the role of digital visualization in choreography, dance performance and documentation" has been explored in dance and technology projects such as Choreographic Morphologies [1]. In their work with prominent choreographers such as Bill T. Jones and Trisha Brown, the OpenEndedGroup have been pioneers in "live visual imagery for motioncaptured dance performance" [15]. They use approaches such as pose recognition and "a distance-mapping-based analysis" of movement, and often employ transparent scrims "allowing the images to share the same space as the performers" [15]. Other projects such as NUVE implement infrared cameras to obtain positional data from the dancer, capturing "the silhouette and the movement of the performer" and allowing for "seamless interaction" between the body of the dancer and the visuals [25]. This enables the dancer to evolve "into a relation with its own 'virtual double' in a way to create a dialog challenging the choreographic boundaries and adding new dimensions to the gesture" [25]. The concept of "virtual body extensions", or interactive visuals as synthetic composites of the performer's moving body, was explored in the project Phantom Limb [6]. A hybrid category of projects, exemplified by Upwell, proposes a combination of installation and performance, allowing "audience members to explore virtual and physical worlds with two dancers" [35].

A related field is the use of interactive visuals for dance 'off-stage': for example, in art installations or laboratorial environments. Fdili Alaoui et al. conducted a study to "design and evaluate interactive systems providing visual feedback that can inform users about their movement qualities" [17]. They analyzed movement qualities from a contemporary dance group and applying it to an installation "designed as an interactive artwork that is used for dance training and education", adopting "interactive visuals based on mass-spring systems" [17]. Brenton et al. have developed and tested (in a lab setting) two prototypes of "interactive visualizations that respond to the free-form movements" of dancers, investigating interactive machine learning as a "methodology for embodied design" [9].

In other cases, interactive visuals have been used with dancers in an 'off-stage' research setting, such as the study by Hsueh et al. (conducted in a dance studio) to explore "the emergent and dynamic relations between the moving body and interactive technology during creative processes such as movement ideation" [21]. The authors "developed a series of interactive visualizations and asked dance practitioners to explore with them" [21], in order to create movement. This led to a taxonomy composed of different relationships and movement responses between dancers and visuals. The taxonomy consists of six different "interaction patterns" organized in two domains: Relationship to Visuals and Movement Type. In the Relationship to Visuals domain, the authors identified three levels (Instrument, Partner and Medium). Instrument refers to manipulation of visual properties by the body, helping dancers form a "first-person relationship"; in Partner, the dancer establishes a dialogue with the visuals, which have an autonomous behavior; and in Medium, visuals are used to communicate with other dancer(s), or between choreographer and dancer. In the Movement Type axis, they propose two levels (Reactive and Self-Reflexive) [21].

Rostami et al. [33] developed five design ideas for interactive performance afforded by bio-sensing and bodily tracking technologies, and use those ideas to discuss three emerging issues: temporality of input, autonomy and control, and visibility of input, in relation to the deployment of bio-sensors and bodily tracking technologies in interactive performances. Although their context is not specifically in dance (their scenarios encompass theatre, street performances, dance, and interactive video performances), their conclusions are relevant for our study, particularly regarding visibility of input. They propose a classification based on a 2 × 2 matrix, with Visibility of Input in the X axis, and Type of interaction (bio-sensing or bodily tracking) in the $\mathrm{Y}$ axis (see Figure 1). 


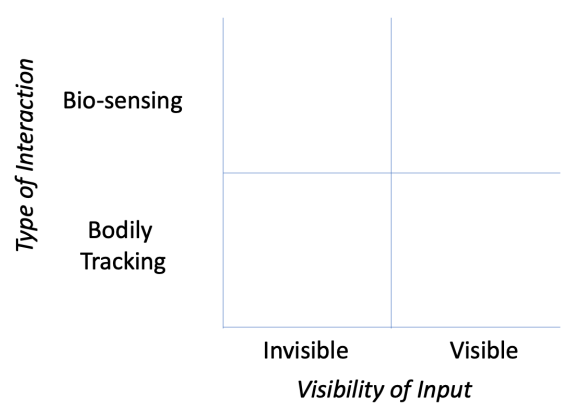

Fig. 1. Visibility of Input matrix, by Rostami et al. [33]

\subsection{Audience Studies in Performance Arts}

There has been an increased interest in audience studies in performance arts involving interactive technology, particularly in electronic music. These studies explore concepts of quality, audience risk and audience experience to redefine performance quality measurement. Bin et al. have developed three relevant studies in this field, analysing: the connection between audience familiarity with a digital musical instrument and enjoyment of a performance [4]; effect of size of gestures, in the interaction with digital musical instruments, on audience enjoyment [3]; and perception of errors in electronic music performances, and how this affects audience enjoyment [5]. Although their research does not involve looking at technological aspects of performance, Radbourne et al. argue that there is a need for research on audience experience as an appropriate and important measure of quality in the performing arts [30]. Their research involved music concerts and theatrical works.

A study by Correia et al. focused on the audience perspective regarding interactive visuals in electronic music performances, 'in the wild' [12]. The results point toward two identified design strategies that could support audience understanding: audiovisual entities and sounding figurations. Capra et al. recently conducted two related studies ('in the lab') regarding interactive visuals as augmentations of the performer's actions. The first a imed to assist audiences to distinguish between human contributions and autonomous processes in electronic music performances. They compared two techniques: pre-performance explanations and visual augmentations. They demonstrate that, unlike pre-performance explanations, "visual augmentations improve the audience experience, increase their subjective comprehension and restore the trust in performers" [11]. The second investigated the impact of varying the level of detail of these visual augmentations on audience experience. In both studies, they combined questionnaires and interviews [10].

Related studies have focused on the element of sound in dance performances. Although we are not analysing sound in the present research, these studies provide an important analogy for us, as they analyse the combination of movement with an additional stimulus (in their case, sound; in our case it will be visuals). Howlin et al. researched how movement and sound combine to produce an audiovisual aesthetics of dance, based on an audience study using video recordings of the original performance [20]. Different audience groups watched three different versions: original, reversed (incongruent) soundtrack or silent. The authors used tablet computers and wrist sensors to collect ratings and physiological data from audience members. The audiovisual incongruent condition was rated as more enjoyable. Reason et al. used qualitative audience research and functional brain imaging (fMRI) to examine the aesthetic experience 
of watching dance both with and without music [31]. The qualitative data revealed that audience members "derived pleasure from finding convergences between two complex stimuli (dance and music)" [31].

\section{METHODS}

\subsection{Audience Studies in Dance Performances with Interactive Technology}

There is a long tradition on audience studies in dance performance, but not specifically in terms of dance and interactive visuals. In this section, we will focus on some studies that also relate methodologically with ours. The project "Watching Dance: Kinesthetic Empathy" [32] used qualitative methods and neuroscience research to analyze how spectators respond to dance, both during and after the performance. Mentis and Johansson employed post-performance interviews with audience members and video analysis to study the audience understanding of movement qualities in an improvised dance performance, involving an element of audience participation [29]. Vukadinović and Marković [39] studied the aesthetic experience of a dance performance from the perspective of both the dancers and the audience. Audience members attended three short custom-made choreographies that were presented live and then watched the recorded versions. They evaluated them on an instrument designed to measure the aesthetic experience of dance. The instrument used semantic differential scales, with 12 seven-point rating scales, across three dimensions (dynamism, exceptionality and affective evaluation). The authors conclude that audiences' aesthetic experience are judged higher when dance choreographies are seen live, compared to recorded versions. Additionally, the specificity of the choreography and of the dancers' expression influences audiences' judgements [39].

\subsection{The Performances}

Previously to the performances, we organized three co-design events in 2019 involving the same group of artists (with some small variations). These events had the following interconnected objectives: 1) the creation of contemporary dance choreographies involving technology, and 2) the development of the technological component to support those choreographies, jointly with our team. The artists were originally selected using an open call, disseminated through mailing lists related to contemporary dance. 92 dancers applied to the call. Ten dance artists were originally selected for the first event, but this was gradually expanded, to the 12 artists participating in the performances presented in this paper (11 female, one male, from seven European countries). We covered travel expenses and paid a fee for each artist. Thanks to the competitive selection, all twelve participants were dance professionals with considerable experience in contemporary dance as performers, some of them also as choreographers. From this group of 12 artists, four took the role of choreographers, of each of the four performances, while the remaining eight took part as dancers.

To conduct our study, we organized an event at Tanzhaus NRW, at a black box theatre. The event was open to the general public and free of cost. In this event, we presented four performances, developed by four different choreographers, in previous stages of our project Moving Digits. Each performance used a different interactive approach to obtain information from the dancers' bodies, in real-time. We will use the term 'interactive condition' to refer to those different approaches (Table 1).

In The Beautiful Glitch, a piece for two dancers, there is an exploration of the limits of motion capture, and the expectations of the audience regarding the reliability of technology. Only one dancer is tracked by the motion capture system (a suit incorporating motion sensors). The visuals include real-time visualizations of the body, created through motion capture data. 
Table 1. Performances presented and interactive technologies adopted

\begin{tabular}{|c|c|}
\hline Performance & Interactive condition \\
\hline 1. The Beautiful Glitch & Mocap suit \\
\hline 2. E-motional Landscapes & $\begin{array}{l}\text { Sensors } \\
\text { (breath, color, positional data) }\end{array}$ \\
\hline 3. Connection Retrieval & Video camera \\
\hline 4. A Dance to Remember & $\begin{array}{l}\text { Minimal interaction } \\
\text { (movement amount) }\end{array}$ \\
\hline
\end{tabular}
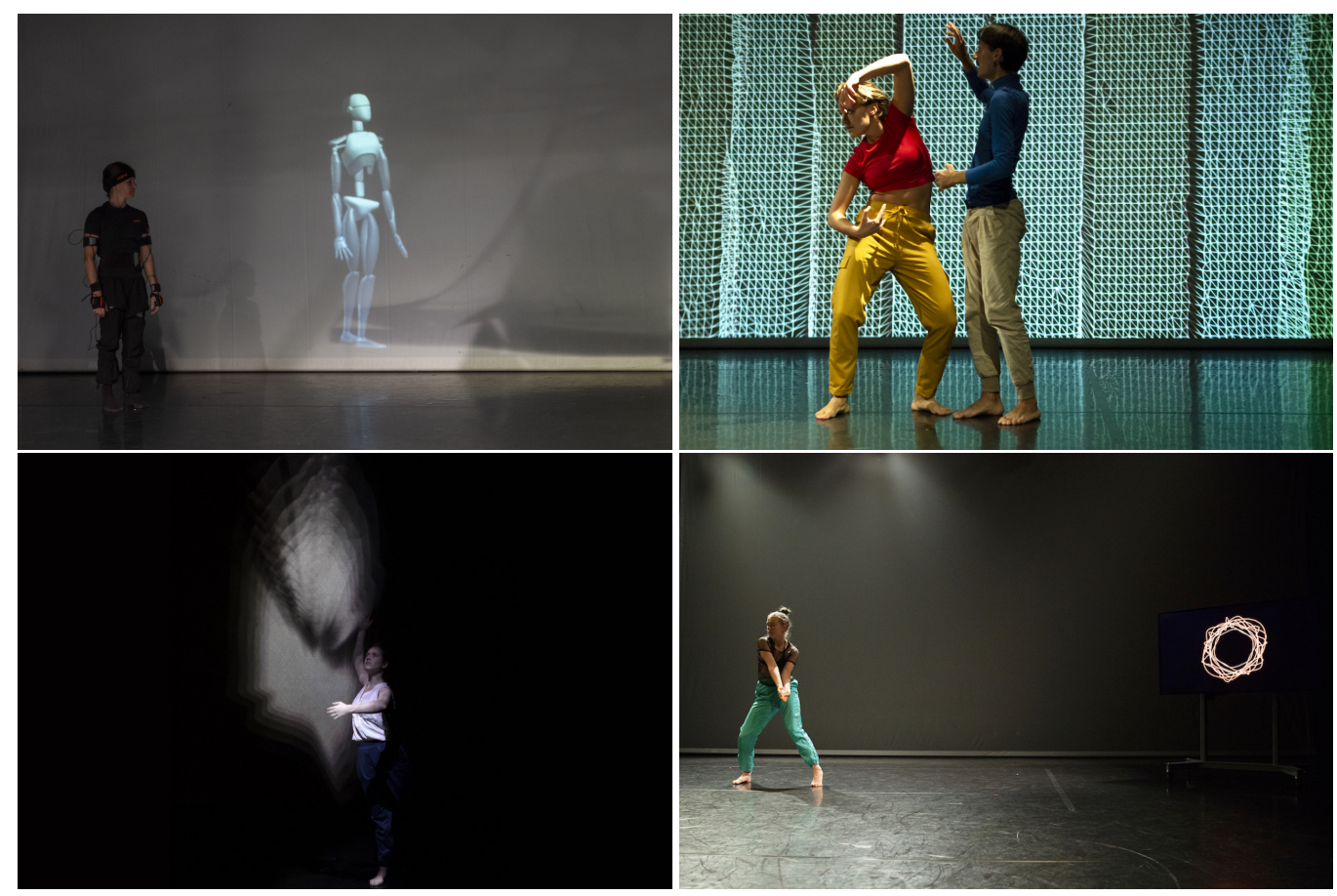

Fig. 2. Photos from the performances. Top: 1. The Beautiful Glitch (left), 2. E-motional Landscapes (right). Bottom: 3. Connection Retrieval (left), 4. A Dance to Remember (right)

E-motional Landscapes explores the relation between the dancer's body and the space. Data from the three dancers is collected based on a breath sensor and their position on stage, tracked by a camera. In another segment, the camera of a mobile phone is used by a dancer as a 'color picker' (the camera is used as a color sensor).

Connection Retrieval explores the dialogue between two dancers, who are either trying to connect or avoid each other. In parts of the piece, one of the dancers is represented through the visuals on the screen, sparking a reaction from the other dancer. In other parts, both dancers are represented on the screen, affecting the movement on stage. In this piece, the camera is used as an input.

A Dance to Remember is a solo piece, and consists of a dialogue between a dancer and an on-screen abstract avatar, that reacts to the movements and attitudes of the dancer and gives her advice. The avatar is actually controlled off- 
stage by the choreographer, creating the illusion of a virtual assistant. There is minimal captured data from the dancer as input - only movement amount of the dancer, tracked via a camera.

In terms of graphic style of visuals, performance 1 used 3D and 2D avatars based on mocap data. Performance 2 processed multiple live camera feeds from the stage, transforming them into geometric, semi-abstract visuals, which were then further manipulated based on sensor data. Performance 3 used video feeds isolating the bodies of the dancers, which were then processed with filters such as blur and twirl. Performance 4 u sed abstract 2D visuals, based on previously prepared frame-by-frame animations, further manipulated (minimally, for example: a slight scaling) taking into account movement amount of the dancer.

Regarding the stage setup of visuals, performances 1 and 2 used a large screen behind the dancers, while performance 3 used a translucent scrim in front of the dancers, and performance 4 used a TV screen. Each performance lasted between 13 and 20 minutes. After each performance, there was a 10-minute break (approximately).

The photos presented in this paper were taken during the event (Figure 2). Videos of each performance, respective text descriptions from the choreographers, and detailed credits, can be found at a dedicated section of the project website $^{2}$. Further detail on the design approaches in performance 1 The Beautiful Glitch can be found in [22] and on performance 3 Connection Retrieval in [28].

\subsection{Participants and Procedure}

The questionnaire was distributed to the audience members at the beginning of the event. The audience was composed of 26 persons (11 female and 15 male, ages ranging between 24 and 62), and all filled in at least partially the questionnaire. At the beginning of the event, we also explained the procedure to the audience: that they should fill in the questionnaire relative to each performance, in the break after each performance. We highlighted that participation was voluntary. After the event, we conducted interviews with four volunteers, previously recruited to be part of the audience and to be interviewed subsequently.

In the questionnaire, we asked two socio-cultural questions, related to frequency of attendance of contemporary dance performances and professional background (in dance; media/design; or none of the above). Regarding the former question, the audience was split almost evenly between occasional attendees and more regular ones: $41 \%$ attend contemporary dance performances very rarely (less than once a year), $5 \%$ attend on average once a year, $36 \%$ attend between once a year and once a month, and $18 \%$ attend once a month or more. Relative to the latter question, $20 \%$ of the respondents work in dance, whereas $48 \%$ work in media/design and $32 \%$ in none of those fields.

\subsection{Questionnaire}

The questionnaire consisted of a demographic section and the following set of questions, repeated for each of the four performances:

(A) Did you perceive a connection between the actions of the dancers and the visuals?

(B) Were the visuals on the screen merely copying the actions of the dancers?

(C) Did the visuals distract you from following the dancers' performance?

(D) Describe with a couple of words the relation between the actions of the dancers and the visuals:

(E) Did you enjoy the visuals in the performance?

(F) Please add a couple of words regarding your opinion on the visuals:

${ }^{2}$ https://movingdigits.eu/c/projects/ 
Questions (D) and (F) were open-ended, the others consisted of 5-point Likert scales, ranging from 1-“strongly disagree" to 5-"strongly agree". The Likert scale questions were informed by the design guidelines in [27] and were analyzed in a previous paper [26] (summarized below). For the present study, we transcribed and analyzed the openended responses, using a combination of word count and manual tagging of the answers, clustered for each performance. For the word count, we used the 'word cruncher' tool in Atlas TI (version 8.4.4) ${ }^{3}$, automatically excluding common words in English.

\subsection{Interviews}

The interviews were semi-structured around the same topics as the questionnaire. The four interviews lasted between 15 and 27 minutes each. They were transcribed and analyzed using thematic analysis [8]. Atlas TI (version 8.4.4) was used to aid in the analysis. Participants had ages between 24 and 62, two male and two female.

\section{RESULTS}

\subsection{Questionnaire - Statistics (Previous Work)}

In our previous paper [26], we analyzed the quantitative component of the questionnaire. We report on the quantitative analysis in this section, in summarized form. 24 persons filled in the Likert scale questions fully. For the statistical analysis, questionnaires partially filled were discarded.

To compare the answers to the four Likert scale questions in the four performances (and hence the respective interactive conditions, as shown in Table 1), we used the Friedman test. As we obtained statistical validity in rejecting the null hypothesis in the four questions, we proceeded with pairwise comparisons. We also performed a Spearman's test to investigate the correlation between each of the first three questions with question (E) enjoyment of visuals. For correlation, we looked at the four performances together. Further detail on the statistical analysis can be found in [26]. We report on comparisons between performances when statistically valid. Table 2 reports the medians of the four questions for the four performances.

Regarding question (A), the result revealed that the video camera interactive condition (performance 3: median = 5) exhibited a more direct connection with the body in comparison to the sensors condition (performance 2: median = 3.5). The video camera condition also had a more direct connection with the body in comparison to mocap suit condition (performance 1: median $=4$ ). No statistical significant difference was found for this question with the minimal interaction condition (performance 4).

Question (B) asked if visuals on the screen were merely copying the actions of the dancers. Visuals in the video camera condition (performance 3: median $=4$ ) were copying the body more in comparison to visuals from the sensors condition (performance 2: median $=2$ ). Visuals in the video camera condition were also copying the body more in comparison to minimal interaction condition (performance 4: median $=1$ ). No statistical significant difference was found for this question with the mocap condition (performance 1). Relatively to question (C), visuals distracting from following the dancers' performance, there was no statistical significance in any pairwise comparison.

Correlation comparing results from question (E) (enjoyment of visuals), showed that the audience enjoys the visuals when a perception of connection between the visuals and the dancers (question $(\mathrm{A})$ ) is established $(\mathrm{r}=0.497)$. The results of comparison with question $(\mathrm{C})$ further indicated that perceived distraction from visuals reduces the enjoyment

${ }^{3}$ https://atlasti.com 
Table 2. Results from the 4 Likert scale questions with the 4 performances, in medians $\left(\left[{ }^{*}\right],\left[{ }^{* *}\right],[\#]\right.$ and $[\# \#$ indicate statistical validity in pairwise comparison)

\begin{tabular}{lcccc}
\hline & \multicolumn{4}{c}{ Performances } \\
Questions & 1 & 2 & 3 & 4 \\
\hline (A) Connection & $4\left[^{*}\right]$ & $3.5\left[^{* *}\right]$ & $5{\left[{ }^{*}\right]\left[{ }^{* *}\right]}^{*} 4$ \\
(B) Copying & 2 & $2[\#]$ & $4[\#][\# \#$ & $1[\# \#]$ \\
(C) Distraction & 3 & 3 & 2.5 & 1 \\
(E) Enjoyment & 4 & 3 & 5 & 3.5 \\
\hline
\end{tabular}

$(\mathrm{r}=-0.349)$. The correlation between visuals merely copying dancers (question (B)) and enjoyment was not statistically significant.

\subsection{Questionnaire - Open-Ended Answers}

25 people answered at least partially to the two open-ended questions: (D) Describe with a couple of words the relation between the actions of the dancers and the visuals; and: (F) Please add a couple of words regarding your opinion on the visuals. The answers of two respondents were not transcribed due to lack of clarity in the hand-writing. 23 respondents answered the questions related to performance 1, 22 respondents for performance 2, 16 respondents for performance 3 , and 18 respondents for performance 4.

We report here the results combining words that appear at least three times with words or sentences particularly relevant to the scope of this paper. Dancers/dance, visual, and body were in general highly used words. To comply with scientific rigour, we report on those words, but we do not always comment on those, as in most of the cases they were just used in the discourse and not as a topic of relevance. We report words from audience replies between quotes.

In performance 1, The Beautiful Glitch, the most used words were: "visual" (7), "dancers" (4), "body" (3), "connection" (3). However, the connection was not always expressed in a positive sense, for instance among the replies, we found "not (...) precise connection" and "disconnected", meaning that the connection was not always present. The visuals were indeed perceived as elements that added information: "I felt that the visuals added information to the purpose of the dancers movement", "I liked the abstract versions and the layers of the visuals". An interaction loop between the dancers and the visuals was also perceived: "I saw the dancers copying the visuals".

In performance 2, E-motional Landscapes, the most used words were: "abstract" (7), "dancers" (5), "visuals" (4), "added" (3). The first word highlights how the abstractness of the visual was the most outstanding component. It is also interesting to note that "added" was quite a present word, meaning that the visuals were adding information or content, for instance: "the visuals were abstract added perspective", "the visuals added different perspectives" and "A 'new' way of communicating an added layer". The theme of visuals as stage design also clearly emerged: "living stage design", and "visuals as stage design", and also it was a "landscape made of humans". On a partially negative side, the connection was not always perceived: "sometimes the connection was very abstract".

In performance 3, Connection Retrieval, the most used words were: "poetic" (5) and "aesthetic" (3). It produced a "fascinating relation" between dancers and visuals. The visuals induced "eye contact between dancers and visuals, direct communication throughout body reaction". In terms of the aesthetic features, the visuals were "breathtaking", but also "ghostly". Poetic is not always used in a positive sense, as in one case the visuals were perceived as "too poetic".

In performance 4, A Dance to Remember, the most used words were: "visuals" (6), "voice" (6), "dialogue" (4), "dancer" (3), "great" (3). The word "conversation" also appeared twice. If we add the four instances of "dialogue" and the two 
instances of "conversation", we can state that this concept was one of the most used. For instance, the technology was "used as a communication tool moving with the rhythm of the computer voice". Moreover, the "relation was well done, very interesting connection". However, some audience members felt that "the visuals seemed less important", with a higher emphasis on voice. This might also be due to the fact that they were not projected but displayed on a TV screen, and one audience member highlighted that "a bigger screen" would have been preferred.

\subsection{Interviews}

In our thematic analysis of the four interviews (interviewees identified as I1-I4), six themes emerged. We report on those below.

4.3.1 Interactivity and mappings. This theme encompasses the participants' perceptions of interactivity between dancers' movements and visuals. This connection was considered obvious in performance 1, noticeable (with some doubts) in performance 2, and more subtle in performance 4. Most participants (I1, I3, I4) promptly recognized the one-to-one mapping through motion tracking in performance 1, which rendered the visuals (composed of avatars) as a direct copy of the actions of the dancers: "it was a copy because it was a one-to-one transmission" (I1).

I2 and I3 noticed a mapping of breath to scaling of visuals in performance 2: "I saw the connection in the sound: the dancer breathed loudly and the graphics scaled down and up accordingly" (I2). I3 considered this to be a positive addition, rather than merely copying the dancers. In this performance, although interactivity was detected, there was a lack of clarity in the mappings: "the connection [between dancers and visuals] is not clear. But I can see that when they move, something happens" (I4). I3 was unable to identify the intentions behind the mapping of colors: "I couldn't clearly see the connection, understand it, because it was all like showing colors and they were wearing colors and I couldn't get what was happening”.

Participants identified some minimal mappings of movement to visual properties, such as scale, shape and color, in performance 4. I2 saw a subtle scaling of visuals, reacting to the dancer's movement: "I had the feeling that the graphics sometimes also got an impulse (...) a scaling" (I2). I2 detected mapping of movement properties to shapes: "the dancer sometimes had very jagged and choppy movements, and the graphics were sometimes more angular and flat"(I2). I4 perceived that there was a "cue" in the visuals, that these were triggered in reaction to the dancer, but that the cue was independent from the dancer: "it's not really based on the dancer's movement" (in fact, it was triggered by the choreographer).

4.3.2 Connection between dancers. This theme highlights the ways in which participants perceive the connection between dancers during performances, assisted by the visuals. This was particularly successful in performance 3 , and considered limiting in performance 1. In performance 1, I1 felt that the visuals "reduced" the movements of the dancers, as one "did nothing independent of the visuals".

Referring to performance 3, I1 felt the connection between the performers as "indirect" and "filtered". She explained how this was made apparent in the performance: "one dancer danced, that it was filtered in some way and changed as a result, and that the other one reacted to it. I thought that was a great, indirect one". It seems that the visual processing ('filtering') of the video image was successful in highlighting the communication, and its qualities, between dancers. Additionally, a kind of "telepresence" effect was evoked through the visuals, which I3 described as "two persons sort of separate, one on the left, one on the right but on this holographic screen, they are all together". A "direct translation of movement" (I3) from one dancer to another through the visuals was also perceived. I1 added that it was the independence of one dancer's movements "that gave it that indirect reaction", and the other dancer "reacted" to the "movement language 
that was translated". She enjoyed this connection and thought that it was "impressive" and "just an aesthetic way to dance".

I1 identified another type of communication in performance 3: "I felt like it was a question-and-answer game (...) later Ifelt that it became more of a conversation and of communicating with each other". I4 felt the connection between dancers and visuals in this performance as "some kind of dialog, (...) connected in a sense the storytelling". This communication between the dancers is further regarded as a "reference" where "only one danced to the other, the other danced for itself (...) provided the template for the reference of the second dancer" (I1). Therefore, the screen provides the template for the second dancer to follow (without the need to look to the first dancer), while allowing some independence to the second dancer.

4.3.3 Power relations. This theme reflects participants' views on the division of power between visuals and dancers. The intensity of visuals ranged from "wasn't too strong", "lagged behind the dancers" (I1, performance 2) to over-powering dancers: "the dancers were not able to take up a position as strong as the visuals had" (I1, performance 1), with performances 3 and 4 achieving a good balance.

Performance 1 brought different reactions from the interviewees. Although I1 considered that the visuals were stronger than the dancers, I4 felt that the power was equally divided between dancers and visuals, which created the illusion of "having like three dancers or four dancers" performing simultaneously. Referring to the power dynamics of performance 3, I1 enjoyed this "three-way division" between the projection and the two dancers, along with the "roles that were distributed with it".

The power relation between visuals and dancers is sometimes experienced as an equal distribution of power, such as in performance 4: "both had their own roles (...) both had their own rooms" (I1), "it was clear from the stage between the two" (I3). The visuals in this performance were seen as embodying their own identity, separated from the dancer. I2 emphasized that the visuals "did not copy the representation", as she saw "the visual level as an individual level". I1 described the power relations between the dancer and visuals as "outstanding": "these two positions [dancer and visuals] also stood for themselves, which means that the dancer had no task to fulfill at all for the visualization".

4.3.4 Aesthetic of visuals. The aesthetic approaches for visuals in performances 2 and 3 were considered successful (with some exceptions), but less so in performances 1 and 4 . Visuals in performance 2 were described as "an abstract digital painting from fragments" (I2), "independent" and "colorful" (I1). Additionally, I2 and I3 referred to the visuals (which occasionally showed the dancers from a top-down point of view) as a presentation of the dancer from a "different perspective, in the form of felt elevation - it's like pulling up a landmass in 3D" (I2), "the top down view was very interesting (...) it was like this perspective shift, because you can see the performance on top point of view" (I3). This "perspective shift" helped I3 "get a perspective on the show that you couldn't normally get", which was "not copying but enhancing", and "adding something to it". However, this approach in performance 2 disoriented I4: "sometimes it gets harder and harder to find where to make my attention".

Participants responded positively to the manipulation of body image in performance 3: "It was very aesthetic for me, and I found it very satisfying to see the dancer, but also this close-up, upscaled more or less directly next to it" (I2). The video effects applied (mentioned as "filter" or "waves" or "distortion") were considered aesthetically pleasing: "one dancer danced, that it was filtered in some way and changed as a result, and that the other one reacted to it" (I1). The visuals in this performance were further described as a image of a "dancer (...) distorted in a wave form" (I2), "independent forms", "some of them were formations, some were wavy lines" (I1), "a hologram with like very visual, trippy, distortion effects" (I3). 
I4 referred to the aesthetic of the visuals as "too direct and (...) kind of flat". He considers that the visuals should be "more complex" as "it feels like they're dancing in front of a camera".

The avatar-based visuals in performance 1 were referred to as "just a basic 3D figure", "not so aesthetic", "very technical (...), very practical" (I2). The figures were considered "abstract and knotted", which was distracting. These visualizations were commented on as merely copying the actions of the dancers (I2). The visuals in performance 4 were unimpressive and "neutral" to I2 and I3, who referred to these visualization as "patterns in the graphic, repetitions, in which nothing more or less new happened at a certain point" (I2) and "a typical sound visualization" (I3).

4.3.5 Added layers and multiple meanings. All participants saw the visuals in the four performances as an added layer, contributing to each performance on top of other elements. I3 compared the glitched visuals in performance 1 to "an error" that was "used in a very artistic way", which made the performance resemble "an artistic visual video". I3 added that the use of glitches in the visuals, combined with one of the dancers trying to replicate those visuals added a "layer of another person copying the other person".

I2 saw the visuals in performance 2, in conjunction with the other elements of the performance (such as colors, costume design and sound), as a "network" of different layers. She enjoyed this "abstract presentation", which allowed to "play with the motion and the colors on these different levels". The visuals "supported [the performance] and added another layer" (I2). This network of different elements building upon each other is again detected in performance 3, where "the dance and the visualization (...) had an aesthetic that also built on one another" (I1). I2 found this network of effects in performance 3 similar to "playing with the different levels", and that it was "appealing" and "beautiful": a "work with light and shadow, and digitally and physically, with different transparencies and different layers".

In performance 4, visuals also contributed to an added layer of meaning, because they were not only used as purely visual material - they were used as language as well (speech and symbolic graphics). In this performance, participants perceived a "meta level" communication (I2), a "dialogue" (I4, I3) between performer and visuals: "there is a connection but on another level (...) like a performer talking to a computer" (I3). I2 similarly saw this interaction as "two individuals who communicated with each other on a meta level". According to I1, the visuals established a "very elaborated visualization language", as the "different symbols included different things" suggesting a kind of "artificial intelligence, which also includes this computer language".

4.3.6 Staging of visuals. The way visuals were set on stage was discussed by participants in performances 3 and 4 . The staging of the dancer and screen (a TV) in performance 4 pleased participants: "I also thought was great that it wasn't somehow everywhere in the room... it also had a frame, it also has its limits" (I1), "this setup made the character of the dancer really strong" (I4).

The results for performance 3 were more ambiguous. I2 described the visuals as "beautiful and appealing", and she enjoyed the way the dancers were placed in regards to the visuals. She felt that the dancing behind the projection in this performance "added another layer", where a direct connection was established between the dancers and the visuals: "through the projection level, both dancers could come into direct contact with each other" (I2). However, I4 considered the "spatially separated" visuals in performance 3 (projected on a translucent screen, filling the right half of the stage) as a distraction: "I was not so sure if I was missing out a lot because of this". Similarly, the distraction resulting from this visual setting was mentioned by I3: "I couldn't decide [where to look], this distracted me a little bit" in performance 3. 


\section{IMPLICATIONS FOR DESIGN}

Taking the results presented above as a starting point, we identify and discuss five design approaches for interactive visuals in dance that can lead to an engaging and aesthetically appealing experience for the audience. All five approaches are based both on open-ended answers to the questionnaire and interviews, with the first two approaches having the additional backing of Likert scale answers to the questionnaire. Therefore, the first two received further attention, leading to a proposed diagram for each. Finally, we also propose how to extend findings to other domains, beyond dance.

\subsection{Balancing Trade-offs Within a Mapping Clarity Spectrum}

The four performances represent different points in a 'mapping clarity spectrum' from the body to the visuals: in one end, clear one-to-one mappings, and in the other end, illegible mappings, with subtle and obscure mappings in between. There is no perfect solution or 'one size fits all' in such creative approaches, but different points in this mapping clarity spectrum offer different trade-offs to the audience, and identifying these from our results is of relevance.

Although direct, one-to-one mappings of data to visuals (such as avatar representations based on mocap data and video images of the body) seem to be more easily recognizable by audience members, they tend to be considered a mere "copy", as exemplified by responses to performance 1 (both in open-ended answers to questionnaire and interviews) and performance 3. Therefore, there is a trade-off between ease of recognition and perception of redundancy by the audience. This trade-off is also supported by the Likert scale answers to the questionnaire, where it was found that performances that registered a stronger connection - between actions of dancers and visuals - were also identified as copying more the body.

Audience members were able to recognize subtle mappings, such as the mapping of a breath sensor to scale of visuals (in performance 2) or of movement amount also to scale of visuals (in performance 4). But with these subtle mappings comes increased ambiguity. In some instances of subtle mappings (performances 2 and 4), there is a recognition of interaction, but audience members are unable to specify what was being mapped. In this case, there is another trade-off: the interaction is adding something new instead of copying what is already on stage, but with an associated risk that the interaction is (excessively) less clear.

This trade-off in subtle mappings relates to ambiguity as a resource for designing interfaces, as a tool for providing a space for interpretation [19], which has been recently explored in the context of electronic music performances [5]. The theme of ambiguity and complex mappings also emerged as a strong need in the use of interactive technology in dance [27]. Interpreting our results in light of the research by Rostami et al. [33], we confirm that all our performances are in the "visible" axis of their visibility of input categorization - aligning with the audience members' recognition of interaction in the performances. Most of them fit within Rostami's "bodily tracking" category (performances 1, 3 and 4), which is mostly classified as "visible", whereas breath sensors (as in performance 2) are classified by Rostami as belonging to the visible axis of the "bio-sensing" category.

Based on these trade-offs regarding mapping clarity, we consider it useful to create a mapping clarity diagram of the performances. This helps situate performances within the interaction design territory they operate, taking legibility for the audience into account. Our starting point was the Visibility of Input classification by Rostami et al. [33], presented in the Related Work section. We propose a new adapted classification, replacing the Visibility of Input axis by a Mapping Clarity axis. Also, as shown by our results, clarity is a continuum, not a discrete binary. We maintained the original Type of Interaction axis, but also removed the boundary between bio-sensing and body tracking, as some performances 


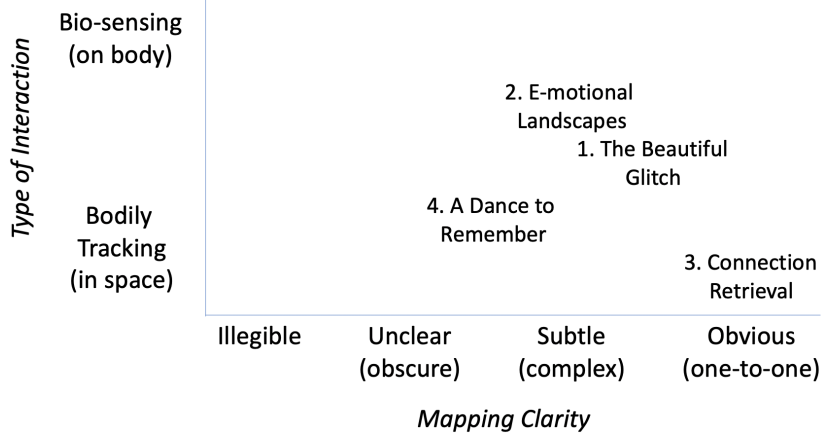

Fig. 3. Mapping Clarity diagram

may combine both (as is the case of performance 2 in our study). We added 'on body' and 'in space' to these Type of Interaction labels, to further clarify this continuum, adopting terminology from Tahiroğlu et al. [36]. The resulting Mapping Clarity diagram is presented in Figure 3.

\subsection{Connecting Layers}

Visuals on stage are naturally an added layer. But for this added layer to be considered well integrated, it is important to connect the visuals thoughtfully to the other elements of the performance. Identifying a connection between visuals and performers was also found to be correlated with enjoyment in the Likert scale answers to the questionnaire. In addition to the connections highlighted above, such as interaction mappings, successful additional strategies (identified in both interviews and open-ended answers to the questionnaire) could be: a) connecting visuals to the set, costume design and sound (as in performance 2); b) harmonize the style of image processing with the choreography and movement qualities (as in performance 3); and c) linking the visuals with more dramaturgical levels of the performance: the story and (eventually) dialogue, using symbolic representation (as in performance 4).

A performance is a constellation of different elements, and our results demonstrate that audiences enjoy recognizing linkages across these elements, from more visible and sensorial, to more conceptual ones (while avoiding redundancy). This relates to the design guidelines for dance and technology suggested by Masu et al. [27], namely: technology should contribute to create multiple layers of meaning, in terms of communication to the audience.

Based on these findings, we argue that, in the multi-layered ecology of a dance performance, interactive visuals have a role to play in not just adding a layer 'on top', but connecting the different layers of the performance, contributing to a holistic and coherent experience. Audience members manifested enjoyment when these connections were detected, and manifested distraction or confusion when they did not. We propose to use the term 'actor' to define both the performers and the visuals, as they are 'liaising' and establishing connections in the multilayered network of the performance. We based this proposal on Latour's Actor-Network theory [24], where actors are not only human individual actors, but they can also be "non-human, non-individual entities", a "source of an action" within a network of multiple dimensions. Based on that, we propose an ecological approach to live visuals, reflected on our Performance Network diagram with connected layers: conceptual layers, on-stage layers and actors (see Figure 4). 


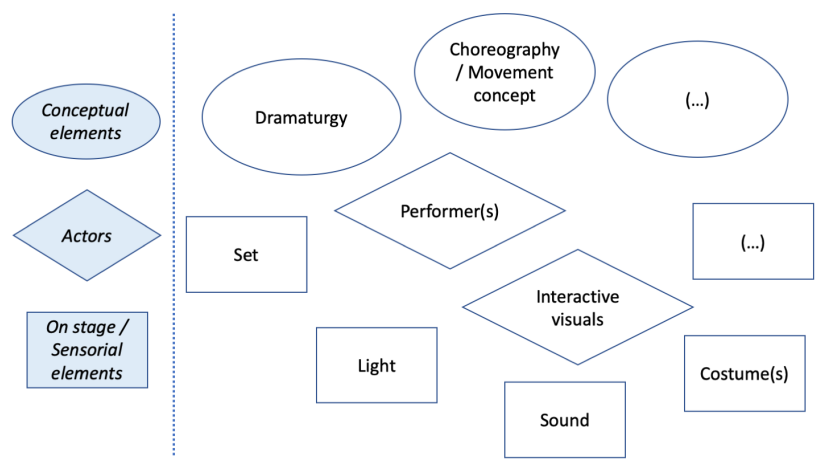

Fig. 4. Performance Network diagram

\subsection{Visuals as Co-Creative Mediator}

Audience members recognized the role of visuals as a mediator between the movement of dancers, based both on open-ended answers to the questionnaire and the interviews. For example, situations where one dancer is reacting to the representation of the other dancer in the visuals, instead of reacting to the other dancer directly (as in performances 1 and 3). However, this mediation can be reductive when it is too prescriptive of movement (as in performance 1). It was considered more successful when the visuals expressed the qualities of the connection between the dancers (and not just mirroring), for example manipulating the visuals of a dancer in a way that conveys the relationship between them (as in performance 3). These results align with the taxonomy by Hsueh et al. [21], namely the Medium level, where visuals are used to communicate with other dancer(s). Within that category, there are two axes: Reactive (directed interaction) and Self-Reflexive (co-creative interaction). Our results suggest that audiences value more the Self-Reflexive axis of co-creative mediation by visuals, rather than a directed one.

\subsection{Defined Territory and Individuality of Visuals}

The stage setup of visuals and dancers can assist in balancing the power between visuals and dancers, so that one does not overpower the other. The two performances that were more successful in this balance ( 3 and 4 ) had a very clear 'territory' for the visuals and for the dancers, while the other two performances used a more 'cinematic' approach of a large, full-width, projection in the back of the stage. These distinct territories, however, should not be stretched too far apart, otherwise there is a risk of leading the audience to feel confused regarding what to focus on (as identified in interviews for performance 3). Scale is also an issue: if the visuals become too small, they may have too little impact (as identified in open-ended answers to questionnaire in performance 4). Another identified successful approach was to convey an individual identity to the visuals, distinct from the dancer (but still reacting to the dancer), as in performance 4. This corresponds to the Partner level in the taxonomy by Hsueh et al., where the visuals have an autonomous behavior [21], and "the virtual body can also be seen as a disembodied image, or a partner". These considerations are supported by the open-ended answers to the questionnaire and the interviews.

\subsection{Exploration of Perspective Shift and Abstract Fragmentation}

The approach of showing the dancers in a different perspective through the visuals, from the one that the audience already sees (as in performance 2), was considered successful (as clearly identified both in interviews and open-ended 
answers to the questionnaire). Another positive strategy regarding visuals was to generate semi-abstract imagery by manipulating camera feeds - a fragmentation of reality (also in performance 2, identified strongly both in interviews and open-ended answers to questionnaire). This double approach of fragmenting reality and shifting perspective relates to cubism in art: to "give the viewer a more accurate understanding of an object, landscape or person by showing it from different angles or viewpoints" and to apply a "breaking down of the real world into flat geometric shapes" [37]. Based on our results, we identify potential in applying cubist principles to interactive visuals.

\subsection{Extending Findings to Other Domains}

We propose that these implications for design can be useful to other fields beyond dance, in contexts where visualizations of embodiment are relevant, such as other artistic fields, games, and broader entertainment areas (e.g. interactive installations, music performances, cultural heritage exhibitions and exergames).

Our insights in section 5.1 Balancing Trade-offs Within a Mapping Clarity Spectrum, and the Mapping Clarity diagram, can assist in achieving a balance in terms of ambiguity of mappings. As Rostami et al. state, there is an "inherent visibility" of the input (bodily tracking tends to be more visible, and bio-sensing less) [33], but we demonstrate that, with visuals, this can be challenged and fine-tuned (e.g. visibility of bodily tracking can be obscured, and bio-sensing data can be highlighted). Regarding section 5.2 Connecting Layers, we emphasize the importance of inter-relating the different elements of the system (both sensorial and conceptual) in terms of design, creating a multi-layered piece (for example, mapping visuals to sound, informed by dramaturgy). Ensuring that both the user and the visuals are in the intersection of these different elements is also key - for example, using some of the strategies we identify in section 5.2. The visuals can act as a unifying 'glue' between the interactions of the user and the other elements in the piece.

Approaches identified in sections 5.3, 5.4 and 5.5 may be useful in specific scenarios: Visuals as Co-Creative Mediator is relevant in multi-user contexts; Defined Territoriality and Individuality of Visuals highlights the importance of framing the visuals in space, as well as the potential for visuals to be an autonomous partner of the user (not only a mirror); and Exploration of Perspective Shift and Abstract Fragmentation can be an important strategy for interactive visuals, allowing to sensorially augment what the user already sees.

\section{CONCLUSION}

In this research, we conducted an audience study 'in the wild', in an event with four different performances combining dance and interactive visuals, aiming to address our research question: What design approaches for interactive visuals in dance can lead to an engaging and aesthetically appealing experience for the audience? The analysis of our study led us to answer this question, by proposing implications for design: 1) Balancing trade-offs within a mapping clarity spectrum; 2) Connecting layers; 3) Visuals as co-creative mediator; 4) Defined territory and individuality of visuals; and 5) Exploration of perspective shift and abstract fragmentation. With these, we hope to assist artists and designers in leveraging interactive technology in performance, fulfilling its potential by making it "accessible to an audience directly" [40]. These implications for design are in dialogue with existing models in this field. We frame our findings within Hsue et al.'s taxonomy of relationships between dancer movements and visuals [21] and adapt Rostami et al.'s Visibility of Input classification [33] into a Mapping Clarity diagram. We also adopt Latour's Actor-Network Theory [24] to develop our own Performance Network diagram, and to highlight the networking role of interactive visuals.

Methodologically, we adopt an 'in the wild' research approach, engaging with "real' users with emerging technologies in 'real' settings under demanding conditions of actual use" [2], with a 'real' audience. We base the study in four heterogeneous performances, by different artists, with disparate interaction and visual design approaches. We use a 
qualitative multi-method approach, based on the analysis of both open-ended responses to questionnaires and interviews - providing, respectively, breadth and depth of data. We contribute to the growing literature on audience studies in technological performance by analysing the impact of interactive visuals in contemporary dance from an audience perspective. In conclusion, we hope that our identified implications for design can be useful to artists, designers and technicians involved in dance, in particular, and other related fields, more generally. We also hope these findings, and our methodological approach, can be of use to researchers in the field of dance studies and embodied interaction.

\section{LIMITATIONS}

Our 'in the wild' methodological approach carries limitations, that we acknowledge: it is more difficult to isolate variables, such as some interactive design decisions, as they are embedded in the idiosyncratic whole of the performance; and there might be biases derived from the order in which performances were presented (it was not possible to randomize and repeat multiple times the event). The small number of interviewees is another limitation for our study. Therefore, we consider that the discussion on implications for design that relies more heavily on questionnaire results than interviews (sections 5.1 and 5.2) can have more general applicability than the remaining part of the discussion (sections 5.3, 5.4 and 5.5). Issues discussed in the latter part of the discussion deserve further research.

\section{ACKNOWLEDGMENTS}

We thank all choreographers and dancers involved, the audience members, the Moving Digits team, and Tanzhaus NRW. This research is co-funded by the Creative Europe programme of the EU (597398-CREA-1-2018-1-PT-CULTCOOP1). We acknowledge the support of LARSyS to this research (Projeto - UIDB/50009/2020). The second author acknowledges ARDITI - Agência Regional para o Desenvolvimento e Tecnologia (project M1420-09-5369-FSE-000002 PhD Studentship).

\section{REFERENCES}

[1] Harry Bailey, James Hewison, and Martin Turner. 2008. Choreographic morphologies: digital visualisation of spatio-temporal structure in dance and the implications for performance and documentations. In Proc. Electronic Visualisation and the Arts (EVA 2008). British Computing Society, London, 9-18. https://ewic.bcs.org/content/ConWebDoc/20578

[2] Steve Benford, Chris Greenhalgh, Andy Crabtree, Martin Flintham, Brendan Walker, Joe Marshall, Boriana Koleva, Stefan Rennick Egglestone, Gabriella Giannachi, Matt Adams, Nick Tandavanitj, and Ju Row Farr. 2013. Performance-Led Research in the Wild. ACM Transactions on Computer-Human Interaction 20, 3 (July 2013), 14:1-14:22. https://doi.org/10.1145/2491500.2491502

[3] S. M. Astrid Bin, Nick Bryan-Kinns, and Andrew McPherson. 2017. Hands where we can see them! Investigating the impact of gesture size on audience perception. In Proc. International Computer Music Conference 2017. ICMC, Shanghai, 1-6. http://hdl.handle.net/2027/spo.bbp2372.2017.052

[4] S. M. Astrid Bin, Nick Bryan-Kinns, and Andrew P. McPherson. 2016. Skip the Pre-Concert Demo: How Technical Familiarity and Musical Style Affect Audience Response. In NIME '16 Proceedings of the 2016 Conference on New Interfaces for Musical Expression. NIME, Brisbane, $200-205$. http://www.nime.org/proceedings/2016/nime2016_paper0041.pdf

[5] S. M. Astrid Bin, Fabio Morreale, Nick Bryan-Kinns, and Andrew P. McPherson. 2017. In-the-moment and Beyond: Combining Post-hoc and Real-Time Data for the Study of Audience Perception of Electronic Music Performance. In Human-Computer Interaction - INTERACT 2017 (Lecture Notes in Computer Science). Springer, Cham, Mumbai, 263-281. https://doi.org/10.1007/978-3-319-67744-6_18

[6] Daniel Bisig and Pablo Palacio. 2014. Phantom Limb - Hybrid Embodiments for Dance. In Proc. XVII Generative Art Conference - GA2014. Generative Art Conference, Milan, 93-107. https://www.generativeart.com/GA2014papers/Daniel_Bisig_Pablo_Palacio_2014.pdf

[7] Andrew Bluff and Andrew Johnston. 2017. Storytelling with Interactive Physical Theatre: A Case Study of Dot and the Kangaroo. In Proceedings of the 4th International Conference on Movement Computing (MOCO '17). ACM, New York, NY, USA, 19:1-19:8. https://doi.org/10.1145/3077981.3078036 event-place: London, United Kingdom.

[8] Virginia Braun and Victoria Clarke. 2006. Using thematic analysis in psychology. Qualitative Research in Psychology 3, 2 (2006), 77-101. https: //doi.org/10.1191/1478088706qp063oa

[9] Harry Brenton, Andrea Kleinsmith, and Marco Gillies. 2014. Embodied Design of Dance Visualisations. In Proceedings of the 2014 International Workshop on Movement and Computing (MOCO '14). ACM, New York, NY, USA, 124:124-124:129. https://doi.org/10.1145/2617995.2618017 event-place: 
Paris, France.

[10] Olivier Capra, Florent Berthaut, and Laurent Grisoni. 2020. All You Need Is LOD : Levels of Detail in Visual Augmentations for the Audience. In The 20th International Conference of New Interfaces for Musical Expression. NIME, Royal Birmingham Conservatoire, United Kingdom, 1-6. https://hal.archives-ouvertes.fr/hal-02560794

[11] Olivier Capra, Florent Berthaut, and Laurent Grisoni. 2020. Have a SEAT on Stage : Restoring Trust with Spectator Experience Augmentation Techniques. In Designing Interactive Systems (DIS). ACM, Eindhoven, Netherlands, 1-13. https://doi.org/10.1145/3357236.3395492

[12] Nuno N. Correia, Deborah Castro, and Atau Tanaka. 2017. The Role of Live Visuals in Audience Understanding of Electronic Music Performances. In Audio Mostly. ACM Press, New York, NY, USA, 1-8. https://doi.org/10.1145/3123514.3123555

[13] Nuno N. Correia and Atau Tanaka. 2014. User-Centered Design of a Tool for Interactive Computer-Generated Audiovisuals. In Proc. 2nd ICLI. ICLI, Lisbon, 1-14. http://www.liveinterfaces.org/proceedings2014.html

[14] Scott deLahunta. 2005. Isadora 'almost out of beta': tracing the development of a new software tool for performing artists. International fournal of Performance Arts and Digital Media 1, 1 (Jan. 2005), 31-46. https://doi.org/10.1386/padm.1.1.31/1

[15] Marc Downie. 2005. Choreographing the extended agent : performance graphics for dance theater. Thesis. Massachusetts Institute of Technology. https://dspace.mit.edu/handle/1721.1/33875

[16] Sarah Fdili Alaoui. 2019. Making an Interactive Dance Piece: Tensions in Integrating Technology in Art. In Proceedings of the 2019 on Designing Interactive Systems Conference (DIS '19). ACM, New York, NY, USA, 1195-1208. https://doi.org/10.1145/3322276.3322289 event-place: San Diego, CA, USA.

[17] Sarah Fdili Alaoui, Frederic Bevilacqua, and Christian Jacquemin. 2015. Interactive Visuals As Metaphors for Dance Movement Qualities. ACM Trans. Interact. Intell. Syst. 5, 3 (Sept. 2015), 13:1-13:24. https://doi.org/10.1145/2738219

[18] Sarah Fdili Alaoui, Christian Jacquemin, and Frédéric Bevilacqua. 2013. Chiseling bodies: an augmented dance performance.. In CHI Extended Abstracts. ACM, Paris, 2915-2918. https://doi.org/10.1145/2468356.2479573

[19] William W. Gaver, Jacob Beaver, and Steve Benford. 2003. Ambiguity as a resource for design. In Proceedings of the SIGCHI Conference on Human Factors in Computing Systems (CHI '03). Association for Computing Machinery, Ft. Lauderdale, Florida, USA, 233-240. https://doi.org/10.1145/642611.642653

[20] Claire Howlin, Staci Vicary, and Guido Orgs. 2018. Audiovisual Aesthetics of Sound and Movement in Contemporary Dance. Empirical Studies of the Arts 38, 2 (Dec. 2018), 0276237418818633. https://doi.org/10.1177/0276237418818633

[21] Stacy Hsueh, Sarah Fdili Alaoui, and Wendy E. Mackay. 2019. Understanding Kinaesthetic Creativity in Dance. In Proceedings of the 2019 CHI Conference on Human Factors in Computing Systems. ACM, Glasgow, 511. https://doi.org/10.1145/3290605.3300741

[22] Stephan Jürgens, Nuno N. Correia, and Raul Masu. 2020. Designing Glitch Procedures and Visualisation Workflows for Markerless Live Motion Capture of Contemporary Dance. In Proceedings of the 7th International Conference on Movement and Computing (MOCO '20). Association for Computing Machinery, Jersey City/Virtual, NJ, USA, 1-8. https://doi.org/10.1145/3401956.3404225

[23] Keina Konno, Richi Owaki, Yoshito Onishi, Ryo Kanda, Sheep, Akiko Takeshita, Tsubasa Nishi, Naoko Shiomi, Kyle McDonald, Satoru Higa, Motoi Shimizu, Yosuke Sakai, Yasuaki Kakehi, Kazuhiro Jo, Yoko Ando, Kazunao Abe, and Takayuki Ito. 2016. Dividual Plays Experimental Lab: An installation derived from Dividual Plays. In Proceedings of the TEI '16: Tenth International Conference on Tangible, Embedded, and Embodied Interaction (TEI '16). Association for Computing Machinery, Eindhoven, Netherlands, 647-652. https://doi.org/10.1145/2839462.2856346

[24] Bruno Latour. 1996. On actor-network theory: A few clarifications. Soziale Welt 47, 4 (1996), 369-381. https://www.jstor.org/stable/40878163 Publisher: Nomos Verlagsgesellschaft mbH.

[25] João Martinho Moura, Né Barros, Pedro Branco, and Adérito Fernandes Marcos. 2010. NUVE: in between the analog and virtual body. In Proceedings of the fifth international conference on Tangible, embedded, and embodied interaction (TEI '11). Association for Computing Machinery, Funchal, Portugal, 409-410. https://doi.org/10.1145/1935701.1935804

[26] Raul Masu and Nuno N. Correia. 2020. Pathways to Live Visuals in Dance Performances: a Quantitative Audience Study. EAI Endorsed Transactions on Creative Technologies 7, 23 (April 2020), 1-8. https://eudl.eu/doi/10.4108/eai.13-7-2018.163987

[27] Raul Masu, Nuno N. Correia, Stephan Jurgens, Ivana Druzetic, and William Primett. 2019. How do Dancers Want to Use Interactive Technology? Appropriation and Layers of Meaning Beyond Traditional Movement Mapping. In Proceedings of the 9th International Conference on Digital and Interactive Arts (ARTECH 2019). Association for Computing Machinery, Braga, Portugal, 1-9. https://doi.org/10.1145/3359852.3359869

[28] Raul Masu, Nuno N. Correia, Stephan Jurgens, Jochen Feitsch, and Teresa Romão. 2020. Designing interactive sonic artefacts for dance performance: an ecological approach. In Proceedings of the 15th International Conference on Audio Mostly (AM '20). ACM, Graz, Austria, 122-129. https: //doi.org/10.1145/3411109.3412297

[29] Helena M. Mentis and Carolina Johansson. 2013. Seeing movement qualities. In Proceedings of the SIGCHI Conference on Human Factors in Computing Systems (CHI '13). Association for Computing Machinery, Paris, France, 3375-3384. https://doi.org/10.1145/2470654.2466462

[30] Jennifer Radbourne, Katya Johanson, Hilary Glow, and Tabitha White. 2009. The Audience Experience: Measuring Quality in the Performing Arts International fournal of Arts Management 11, 3 (2009), 16-29. http://www.jstor.org/stable/41064995http://about.jstor.org/terms

[31] Matthew Reason, Corinne Jola, Rosie Kay, Dee Reynolds, Jukka-Pekka Kauppi, Marie-Helene Grobras, Jussi Tohka, and Frank E. Pollick. 2016. Spectators' aesthetic experience of sound and movement in dance performance: A transdisciplinary investigation. Psychology of Aesthetics, Creativity and the Arts 10, 1 (2016), 42-55. https://doi.org/10.1037/a0040032 Place: US Publisher: Educational Publishing Foundation.

[32] Dee Reynolds. 2011. Watching Dance: Kinesthetic Empathy. http://www.watchingdance.org/ 
[33] Asreen Rostami, Donald McMillan, Elena Márquez Segura, Chiara Rossito, and Louise Barkhuus. 2017. Bio-Sensed and Embodied Participation in Interactive Performance. In Proceedings of the Eleventh International Conference on Tangible, Embedded, and Embodied Interaction (TEI '17). Association for Computing Machinery, Yokohama, Japan, 197-208. https://doi.org/10.1145/3024969.3024998

[34] Chris Salter. 2010. Entangled: Technology and the Transformation of Performance. MIT Press, Massachusetts.

[35] Jinsil Hwaryoung Seo, Michael Bruner, Nathan Ayres, Christine Bergeron, and Alexandra Pooley. 2019. Upwell: Performative Immersion Hybridizing Two Worlds. In Proceedings of the Thirteenth International Conference on Tangible, Embedded, and Embodied Interaction (TEI '19). Association for Computing Machinery, Tempe, Arizona, USA, 571-575. https://doi.org/10.1145/3294109.3301264

[36] Koray Tahiroğlu, Nuno N. Correia, and Miguel Espada. 2013. PESI Extended System: In Space, On Body, with 3 Musicians. In Proceedings of New Interfaces for Musical Expression (NIME). NIME, Daejeon, Seoul, 35-40. https://zenodo.org/record/1178666

[37] Tate. [n.d.]. All about cubism. https://www.tate.org.uk/art/art-terms/c/cubism/all-about-cubism Library Catalog: www.tate.org.uk Section: Art \& artists.

[38] Peter A. Torpey. 2013. Media Scores: A Framework for Composing the Modern-Day Gesamtkunstwerk. PhD thesis. MIT, Cambridge, MA. https: //www.media.mit.edu/publications/media-scores-a-framework-for-composing-the-modern-day-gesamtkunstwerk/

[39] Maja S. Vukadinović and Slobodan Marković. 2017. The relationship between the dancers' and the audience's aesthetic experience. Psihologija 50, 4 (2017), 465-481. http://www.doiserbia.nb.rs/Article.aspx?ID=0048-57051700009V

[40] Robert Wechsler, Frieder Weiß, and Peter Dowling. 2004. EyeCon - a motion sensing tool for creating interactive dance, music and video projections. In Proceedings of the Society for the Study of Artificial Intelligence and the Simulation of Behavior (SSAISB)'s convention: Motion, Emotion and Cognition. Society for the Study of Artificial Intelligence and the Simulation of Behavior, Leeds, 1-7. http://www.palindrome.de/content/pubs/leeds.pdf 